PAPER • OPEN ACCESS

\title{
The Strip SiPM: a study of single photon time resolution
}

To cite this article: K. Doroud et al 2021 JINST 16 P06017

View the article online for updates and enhancements.

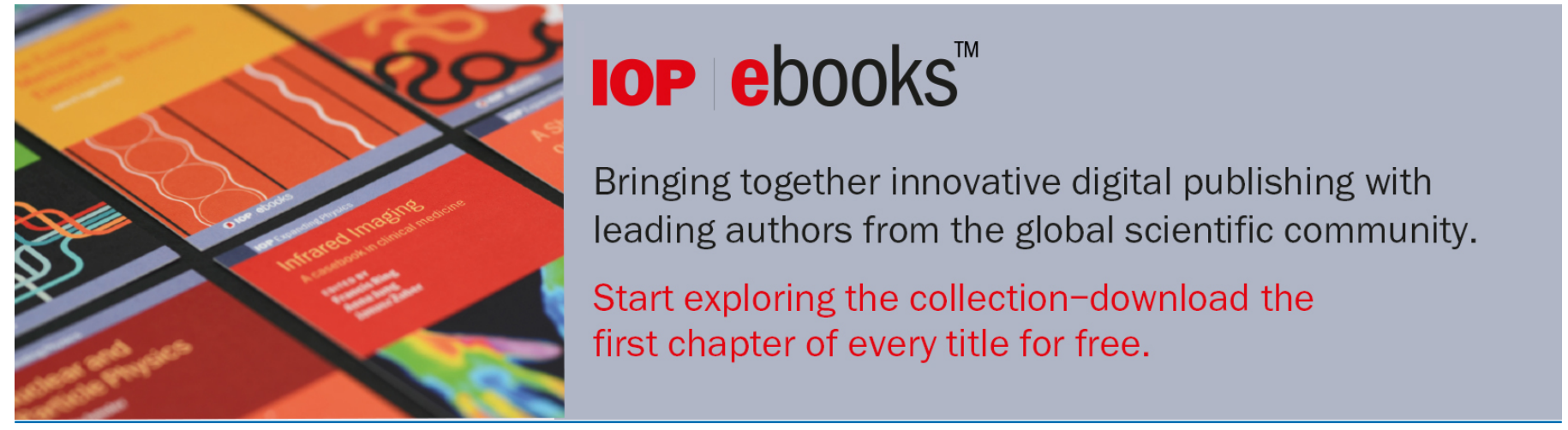

This content was downloaded from IP address 128.141 .192 .28 on 21/07/2021 at 16:43 


\title{
The Strip SiPM: a study of single photon time resolution
}

\author{
K. Doroud,${ }^{a, b, c}$ D.W. Kim, ${ }^{c}$ K.H. Kwon, ${ }^{c}$ Z. Liu, ${ }^{d, *}$ B.G. Min, ${ }^{c}$ M.C.S. Williams ${ }^{a, c, e}$ \\ and A. Zichichi ${ }^{a, e, f}$ \\ ${ }^{a}$ European Centre for Nuclear Research (CERN), Geneva, Switzerland \\ ${ }^{b}$ ICSC World Laboratory, Geneva, Switzerland \\ ${ }^{c}$ Gangneung-Wonju National University, Gangneung, South Korea \\ ${ }^{d}$ Paul C. Lauterbur Research Centre for Biomedical Imaging, \\ Shenzhen Institutes of Advanced Technology, Chinese Academy of Sciences, Shenzhen 518055, China \\ ${ }^{e}$ INFN and Dipartimento di Fisica e Astronomia, Università di Bologna, Italy \\ ${ }^{f}$ Museo Storico della Fisica e Centro Studi e Ricerche E. Fermi, Roma, Italy \\ E-mail: Zheng.Liu@siat.ac.cn
}

\begin{abstract}
The Strip Silicon PhotoMultiplier (SiPM) allows a photosensitive area to be created that has excellent time resolution coupled to good position resolution. However, as the SiPM is made larger it becomes more difficult to extract the signal produced by a single photon; we thus are using the NINO ASIC as the front-end amplifier. The NINO ASIC dissipates $40 \mathrm{~mW}$ of heat per channel. SiPMs are sensitive to temperature; increasing the temperature leads to higher noise and a higher breakdown voltage. For the best timing performance the front-end electronics should be mounted as close to the SiPM as possible; however if, for example, the front-end electronics are mounted on the backside of the SiPM, it would be very difficult to control the temperature of the SiPM. Given this, we chose to mount the NINO ASIC on the printed circuit board (PCB) away from the strip SiPM so that a cooling system can be added. We present here the performance of an array of 16 strip SiPMs readout by 4 NINO ASICs coupled by $\sim 5 \mathrm{~cm}$ of PCB track length. The performance has been evaluated by the measurement of the Single Photon Time Resolution (SPTR) and also the position resolution along the strip determined from the time difference of the signals at each end.
\end{abstract}

Keywords: Photon detectors for UV, visible and IR photons (solid-state); Photon detectors for UV, visible and IR photons (solid-state) (PIN diodes, APDs, Si-PMTs, G-APDs, CCDs, EBCCDs, EMCCDs, CMOS imagers, etc)

\footnotetext{
${ }^{*}$ Corresponding author.
} 


\section{Contents}

1 Introduction 1

2 Experimental setup $\quad 4$

3 Results 4

4 Discussion $\quad 8$

\section{Introduction}

The Silicon PhotoMultiplier (SiPM), a solid-state photon counting detector, has been developed through the pioneering work of V. Golovin [1] and Z. Sadygov [2]. The device consists of an array of small avalanche photo diodes run in Gieger mode. A single photoelectron can cause a Geiger breakdown in an individual photo diode and create a detectable signal, thus these photo diodes are known as Single Photon Avalanche Diodes (SPAD). Even though the signal from a single SPAD is sufficiently large such that single photons can be detected when the array of SPADs is large; the fact is that as the array becomes larger, the signal of an individual SPAD firing is degraded (mostly due to the increased capacitance of the SiPM device) making single photon detection and timing difficult. An important step forward was made by fabricating a trench around each SPAD; this substantially reduces the probability that a firing SPAD will also fire a neighbouring SPAD (i.e.crosstalk) and allows the SiPM to be operated at much higher voltages (generating bigger signals) [3] with a reduction of crosstalk. When many SiPM devices are gathered together to make a 'multi-cell' device, care has to be taken so that noise from neighbouring devices does not degrade the performance normally obtained by a single device operated alone. Differential readout is an important aspect when precise timing is required [4].

The Strip Silicon PhotoMultilier (SiPM) is a detector configuration that allows a large photosensitive area to be created with excellent time and position resolution [5]. Both anode and cathode are read out at each end; this differential read out results in a reduction of electronic noise and cross-talk. The strip SiPM array discussed in this paper differ from those reported in reference [5] as they are $1 \mathrm{~mm}$ wide instead of $0.5 \mathrm{~mm}$ wide. The array consists of 16 strips, each having a length of $18 \mathrm{~mm}$ long as shown in figure 1 . Each strip is manufactured ${ }^{1}$ individually and mounted on a small PCB; the minimum spacing between strips that can be guaranteed is $80 \mu \mathrm{m}$; in this particular case the strips are clustered in groups of four with $250 \mu \mathrm{m}$ between each group since they are destined to read out an array of crystals. The size of the SPAD is $50 \times 50 \mu \mathrm{m}^{2}$ similar in structure to the S13361series of MPPCs made by Hamamatsu. An importance difference between the strip

${ }^{1}$ These strip SiPMs have been manufactured by Hamamatsu Photonics K.K. (HPK); Hamamatsu use the name MultiPixel Photon Counters (MPPC) for their products but we will refer to them with their more common name of SiPM in this paper. 
Area of each strip is $18 \mathrm{~mm} \times 1 \mathrm{~mm}$

80 micron space between each strip 250 micron space between every group of four strips

\begin{tabular}{|c|c|c|}
\hline $\begin{array}{l}\text { A1a } \\
\text { K1a }\end{array}$ & strip 16 & $\begin{array}{l}\text { K1b } \\
\text { 口 A1b }^{2}\end{array}$ \\
\hline $\begin{array}{l}\text { A2a } \\
\text { K2a }\end{array}$ & strip 15 & $\begin{array}{l}\text { K.2b } \\
\square \text { A2b }\end{array}$ \\
\hline $\begin{array}{l}\text { A1a } \\
\text { K3a }\end{array}$ & strip 14 & $\begin{array}{l}\text { 口 K3b } \\
\text { 口A1b }\end{array}$ \\
\hline $\begin{array}{l}\text { A4a 口 } \\
\text { K4a } \square\end{array}$ & strip 13 & $\begin{array}{l}\text { ㅈb } \\
\text { 口A4b }\end{array}$ \\
\hline 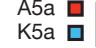 & strip 12 & $\begin{array}{l}\text { ․ K5b } \\
\text { 口A5b }\end{array}$ \\
\hline $\begin{array}{l}\text { A6a } \\
\text { K6a } \square\end{array}$ & strip 11 & $\begin{array}{l}\text { ㅁ6b } \\
\text { 口A6b }\end{array}$ \\
\hline $\begin{array}{l}\text { A7a 口 } \\
\text { K7a } \square\end{array}$ & strip 10 & $\begin{array}{l}\text { GK7b } \\
\text { a A7b }\end{array}$ \\
\hline 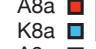 & strip 9 & $\begin{array}{l}\text { G8b } \\
\text { QA8b }\end{array}$ \\
\hline $\begin{array}{l}\text { A9a } \\
\text { K9a }\end{array}$ & strip 8 & $\begin{array}{l}\text { ㅁ9b } \\
\text { 口A9b }\end{array}$ \\
\hline $\begin{array}{l}\text { A10a 口 } \\
\text { K10a } \square\end{array}$ & strip 7 & $\begin{array}{l}\text { Q K10b } \\
\text { a10b }\end{array}$ \\
\hline $\begin{array}{l}\text { A11a } \\
\text { K11a } \square\end{array}$ & strip 6 & $\begin{array}{l}\text { 口K11b } \\
\text { QA11b }\end{array}$ \\
\hline $\begin{array}{l}\text { A12a } \\
\text { K12a }\end{array}$ & strip 5 & $\begin{array}{l}\text { G12b } \\
\text { GA12b }\end{array}$ \\
\hline $\begin{array}{l}\text { A13a } \square \\
\text { K13a }\end{array}$ & strip 4 & $\begin{array}{l}\text { 口K13b } \\
\text { 口A13b }\end{array}$ \\
\hline $\begin{array}{l}\text { A14a 口 } \\
\text { K14a } \square\end{array}$ & strip 3 & $\begin{array}{l}\text { 口K14b } \\
\text { 口A14b }\end{array}$ \\
\hline $\begin{array}{l}\text { A15a 口 } \\
\text { K15a } \square\end{array}$ & strip 2 & $\begin{array}{l}\text { 口K15b } \\
\text { 口A15b }\end{array}$ \\
\hline $\begin{array}{l}\text { A16a ㅁ } \\
\text { K16a } \mathrm{口}\end{array}$ & strip 1 & $\begin{array}{l}\text { K16b } \\
\text { 口 }^{A 16 b}\end{array}$ \\
\hline
\end{tabular}

Figure 1. The 16 strip SiPM array: the overall active area is $18 \times 17.7 \mathrm{~mm}^{2}$.

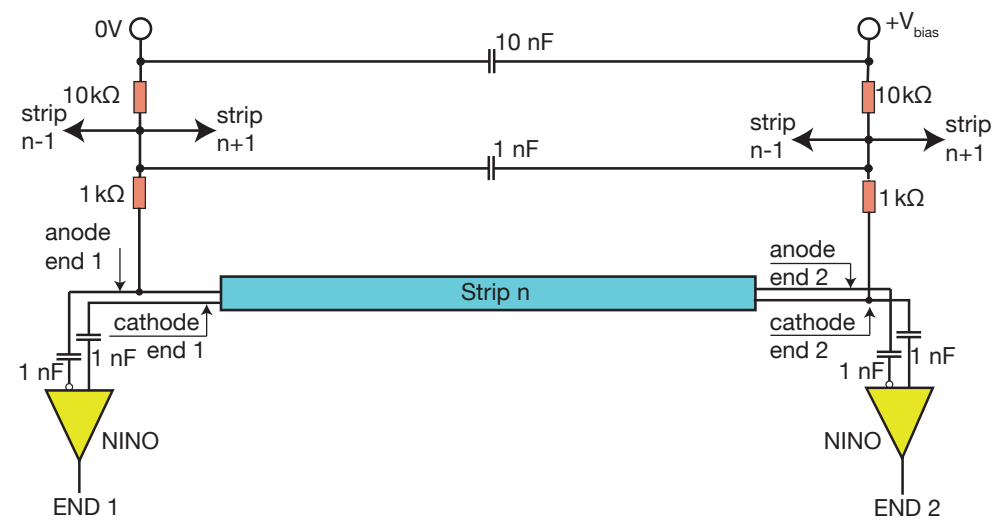

Figure 2. Circuit diagram showing biasing and read-out of a single strip SiPM.

SiPM and standard square SiPMs is that the Aluminium grid on the top surface is made thicker and the 'backside' of the strip is aluminised. This is to reduce the DC resistance as much as possible so that the strip acts as a transmission line.

SiPMs are very sensitive to temperature; the breakdown voltage and dark noise vary with temperature [6]. We use the Hamamatsu C14156 MPPC power bias supply to generate the voltage for all 16 strips of the SiPM array; the $\mathrm{C} 14156$ incorporates a precision voltage adjustment controlled by a thermistor that is mounted close to the SiPM array to sense the temperature. The NINO ASIC [7] is used to read out the strip SiPM; the circuit diagram corresponding to the readout of one strip is shown in figure 2. Each channel of NINO generates $40 \mathrm{~mW}$ of heat; thus the 32 channels needed to 


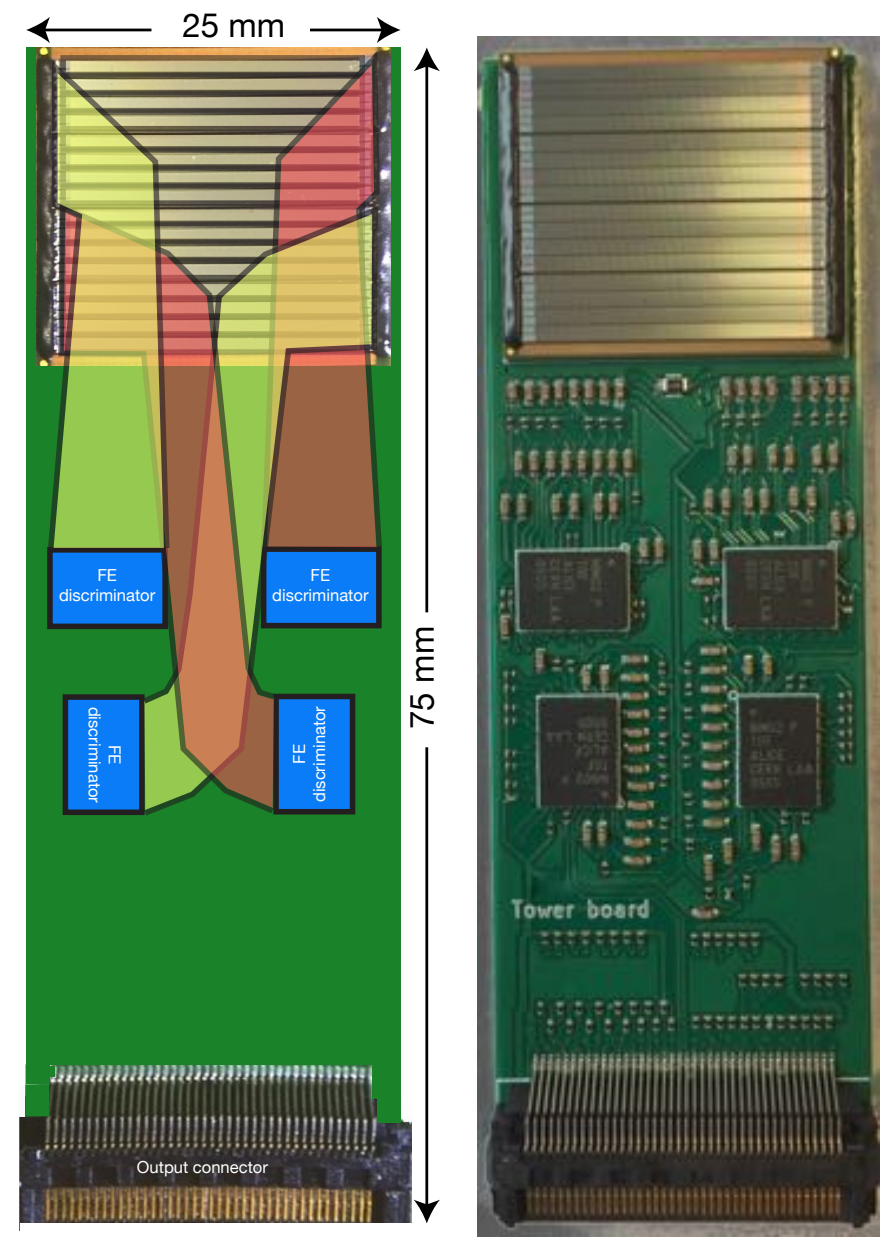

Figure 3. Each end of the strip SiPMs need to be connected to the amplifier/discriminator with a transmission line. This shows the schematic arrangement of the transmission lines. These lines need to be short so that the signals do not degrade too much - however the amplifier/discriminator needs to be moved away from the SiPMs so that the temperature of the SiPMs can be controlled. A photo of the actual card is shown on the right.

readout the 16 strip SiPM array will generate $1.3 \mathrm{~W}$ of heat. Given this, we need to move the NINO ASICs away from the strip SiPMs so that cooling can be added to keep the Strip SiPMs at a constant temperature $\left(18^{\circ} \mathrm{C}\right.$ in this case). This is illustrated in figure 3 where the PCB board is shown. We have chosen the paths of the transmission lines bringing the signals to the NINO input to follow the layout shown in figure 3; this scheme allows the length of each transmission line to be roughly equal. The transmission line between the SiPM and the input of the NINO is tuned to be $50 \Omega$ (the input impedance of the NINO); ground layers separate the layers containing the transmission lines. The PCB is a 10 layer board with an overall thickness of $1.6 \mathrm{~mm}$.

The layout of the PCB presented above (with the front-end asics separated from the array of Strip SiPMs) was designed with the readout of scintillating crystals in mind. A schematic drawing of the cross section of the proposed design is shown in figure 4. The NINO ASICs are coupled with thermal grease to a water-cooled aluminium block. Alternative designs can be considered such as 


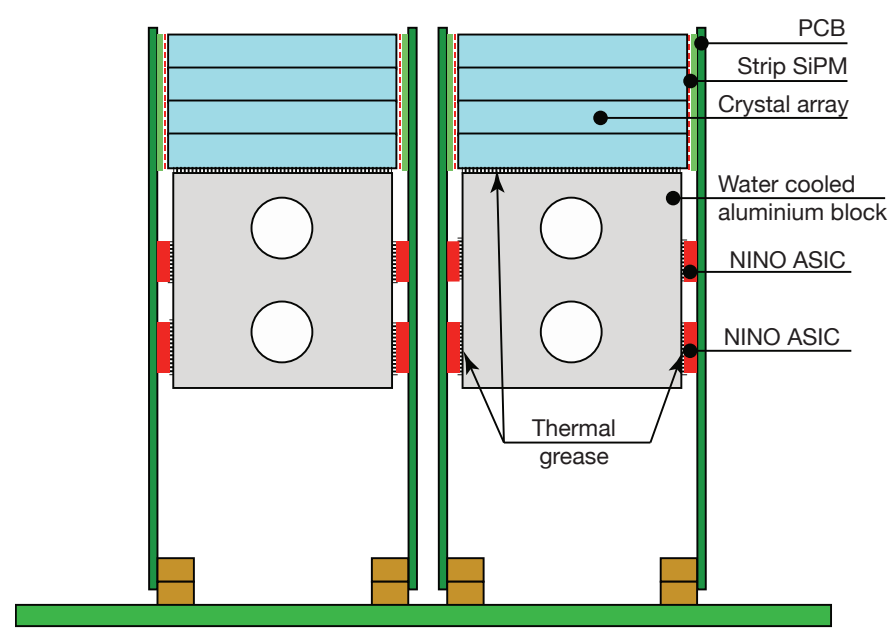

Figure 4. Schematic diagram showing a possible cooling system in the case that an matrix of scintillating crystals are read out by two arrays of strip SiPM. Water is flowed through the aluminium block that is thermally coupled to the surface of the NINO ASICs; this aluminium block is also thermally coupled to the matrix of scintillating crystals that are glued to the Strip SiPM matrices.

mounting the front-end electronics on the backside of the strip SiPMs (thus keeping the connections between the SiPM and the amplifier-discriminator as short as possible); however such a scheme would lead to heating of the SiPMs unless a cooling medium was flowed within the PCB itself.

\section{Experimental setup}

The strip SiPM card was mounted in a cooled dark box; the cooling was provided by water flowing through a radiator with fans to circulate the air. The temperature within the box was monitored and was generally in the range $17-18^{\circ} \mathrm{C}$. A laser ${ }^{2}$ with a wavelength of $405 \mathrm{~nm}$ is used for the measurement; optical filters were used to reduce the light intensity such that only $30 \%$ of the laser pulses have a corresponding SiPM event; thus if the SiPM fires, it will usually be a single photo-electron event [9]. The outputs from the NINO ASICs are routed with twisted pair cables to 32 channels of a 64 channel SAMPET module; the SAMPET is a version of the SAMPIC [8] but designed to receive a differential logic and measure the time of the leading edge and width of input signal. This system makes timing measurements of the leading edge with a resolution of $7 \mathrm{ps} \sigma$ together with a pulse width measurement (i.e. TOT) with a precision better than $1 \mathrm{~ns}$.

\section{Results}

Figure 5 shows the current drawn by the array of 16 strip SiPMs versus the applied reverse bias voltage; this indicates that the breakdown voltage is around $52 \mathrm{~V}$. A critical parameter for SiPMs is the noise rate. With a Strip SiPM, the time difference between the signals arriving at each end can be used to determine where along the length of the strip the noise pulse originated. Ideally all positions along the strip are equally probable, thus the time difference spectrum from a given strip

\footnotetext{
${ }^{2}$ The laser was a PiL040X with a EIG2000DX controller.
} 


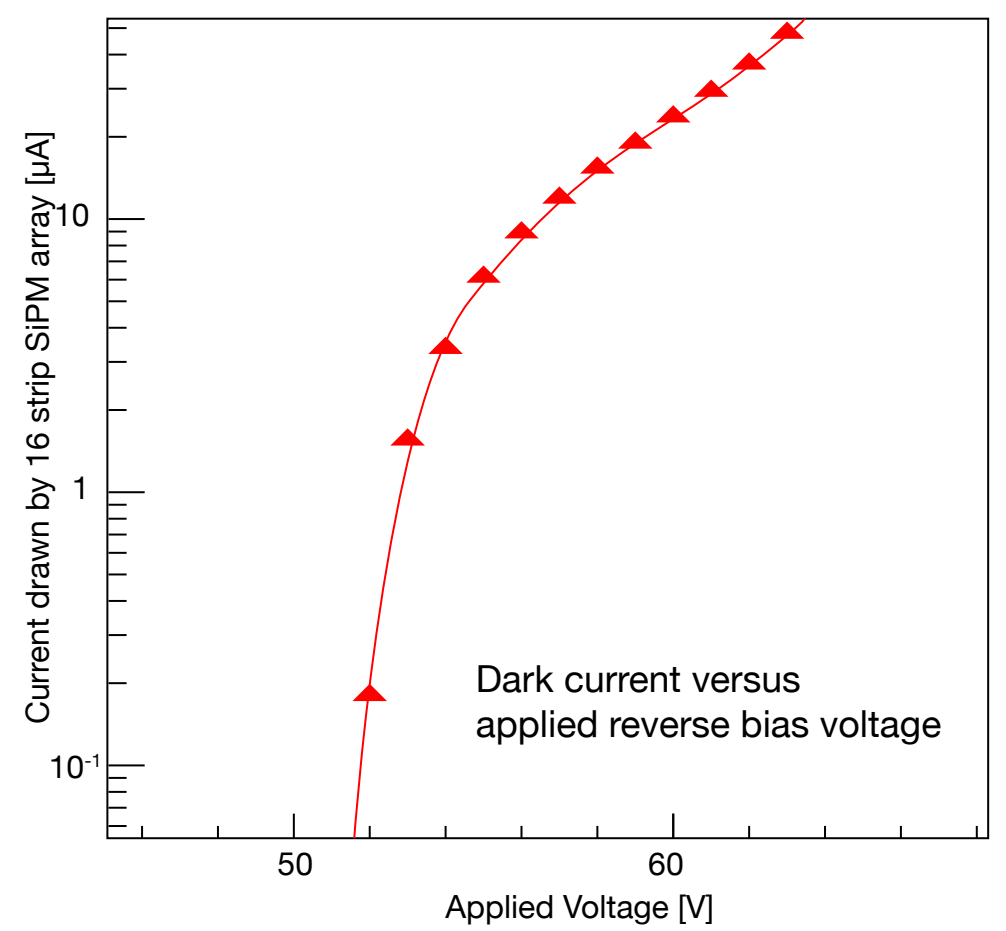

Figure 5. Dark current drawn by 16 strip SiPM as a function of applied reverse-bias voltage. This data was taken at $18^{\circ} \mathrm{C}$.

should be in the form of a box. The results of a run taken at $61 \mathrm{~V}$ and with the NINO threshold set to $80 \mathrm{mV}^{3}$ are shown in figure 6 . The box shape indicates that the noise is homogeneous along the length of the strip; the similar number of events within each of the boxes indicates similar noise in all strips; the edges of the box distribution give an indication of the position resolution that can be obtained by using the time difference from each end. Typically at the edge passes the $25 \%$ to $75 \%$ in 4 bins of 25 ps (i.e. $100 \mathrm{ps}$ ) for a total width of the box of $1.4 \mathrm{~ns}$. As the strip is $18 \mathrm{~mm}$ long this indicates that the FWHM position resolution is $1.3 \mathrm{~mm}$. A more precise measurement where a laser is used to measure selected positions along the length of the strip is presented below.

The NINO ASIC measures the input charge by a time-over-threshold (TOT) technique such that the width of the output logic signal is related to the input charge. Figure 7 shows a typical TOT spectrum measured at one end of the strip when the strip SiPM is illuminated with laser. The peaks in this spectrum correspond to one and two photo-electrons (two photo-electron events are generated either when two photons within a laser pulse are detected, or when a neighbouring SPAD is fired due to cross-talk [10]). Single photo-electrons events were selected to be within the Full-Width-Half-Max (FWHM) of the single photo-electron peak (shown on figure 7). Although this cut removes a number of single photo-electron events, it did not affect the measurement of the single photon time resolution or the late tail discussed below.

Figure 8 shows the dark count rate (DCR) versus the applied threshold for a variety of applied bias voltages to the strip. The rapid decrease in the DCR occurs when the threshold is increased

${ }^{3}$ All NINO threshold values quoted in this paper refer to the voltage difference between the threshold inputs (+ and -) on the NINO ASIC. 

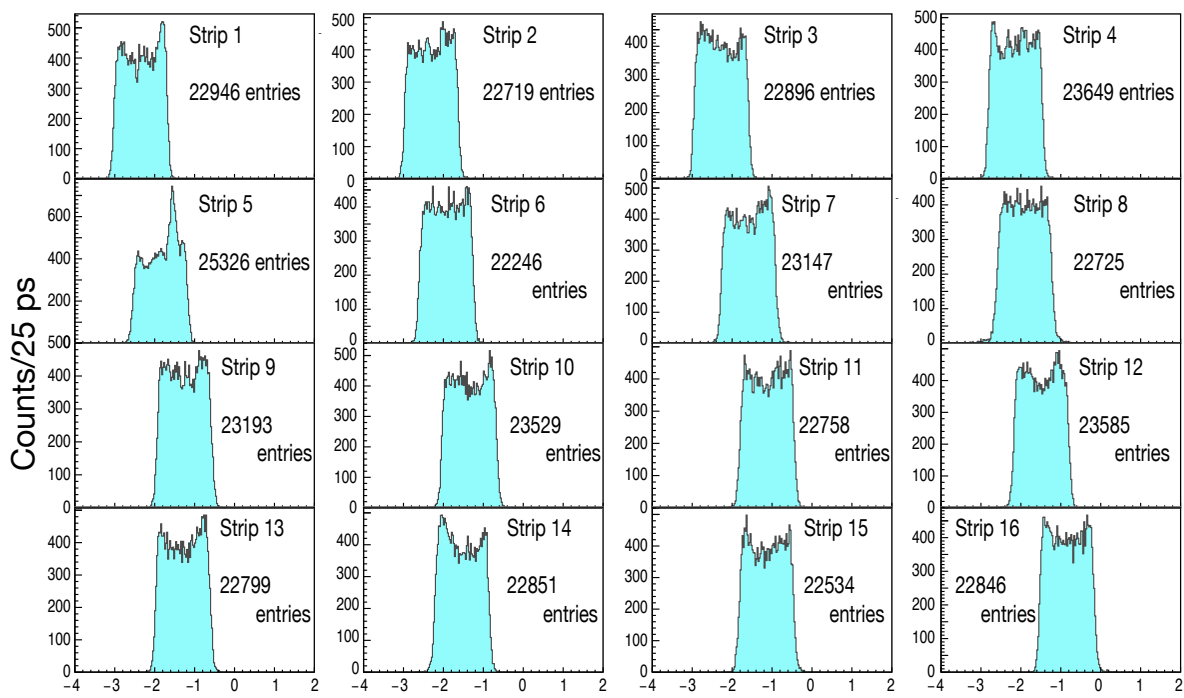

Time difference between the two ends of the strips [ns]

Figure 6. Noise counts (dark count rate (DCR)) for the 16 strips SiPM. The time difference between the two ends is shown and should ideally be a box that corresponds to the length of the strip.

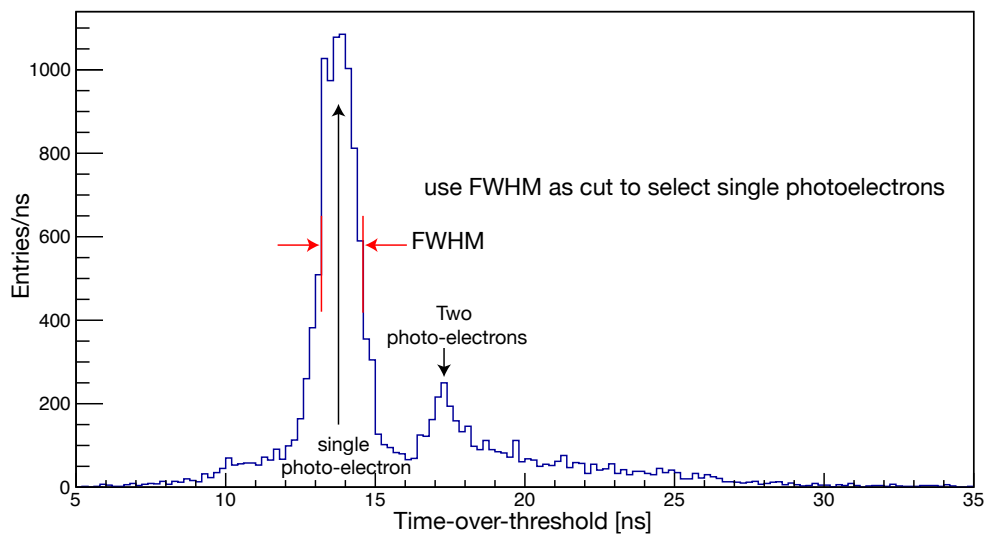

Figure 7. Typical time-over-threshold spectrum: the peak of the single photo-electron signal was determined - and then the full width half maximum (FWHM) determined. The FWHM was used to automatically define cuts to select single photo-electron events.

above the signal produced when just one SPAD fires. Data presented in this paper were taken at a NINO threshold of $80 \mathrm{mV}$ unless otherwise stated.

The signals from each end of the strip SiPM were discriminated by NINO as shown in figure 2 and digitised by the SAMPET module. The average of the time from each end was compared with the time that the laser was triggered. This, with the single photo-electron selection discussed in figure 7, gives the single photon time resolution (SPTR). The result is shown in figure 9. It should be noted that this SPTR spectrum has a late tail. If the Gaussian fit is limited to exclude this tail then the $\sigma$ of the fit is reduced (this is shown in the inset in figure 9). All time resolutions quoted in this paper are from a fit to the full time spectrum (i.e. the late tail is always included).

The laser was mounted on a moving stage so that the laser could be positioned such that the laser light was focused at the centre of one of the strips making the array of 16. The spot of the laser 


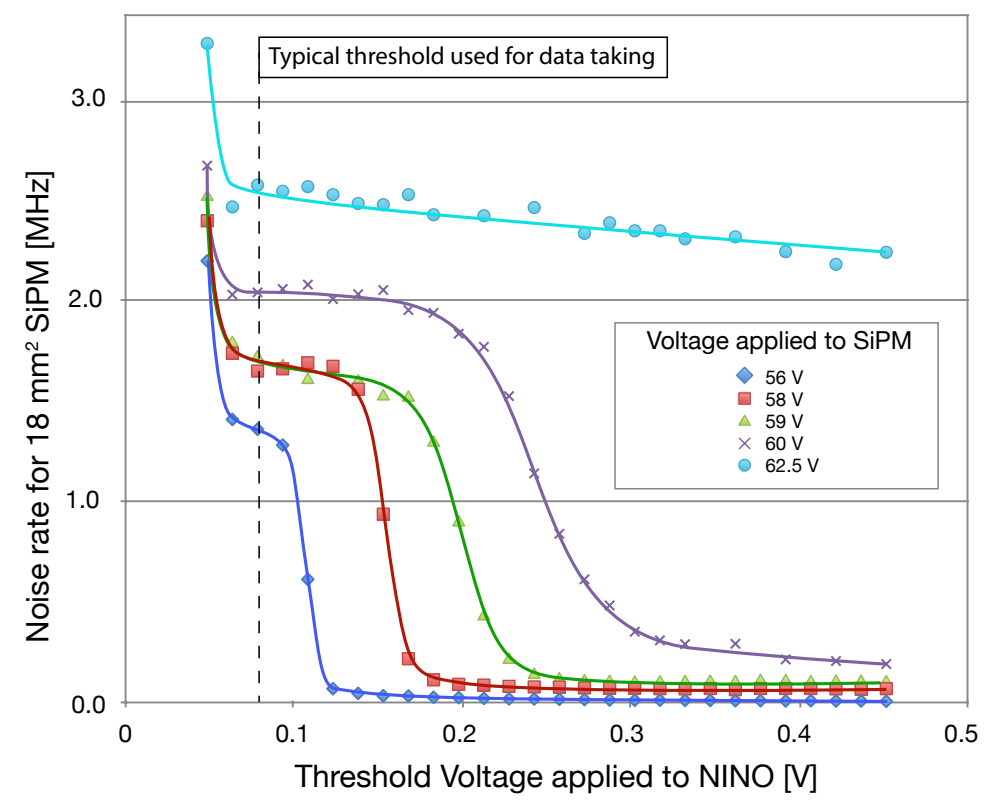

Figure 8. Dark noise count versus threshold voltage for a variety of bias voltage applied to the SiPM. The data presented in this paper were taken with a voltage of $0.8 \mathrm{~V}$ across the NINO threshold control.

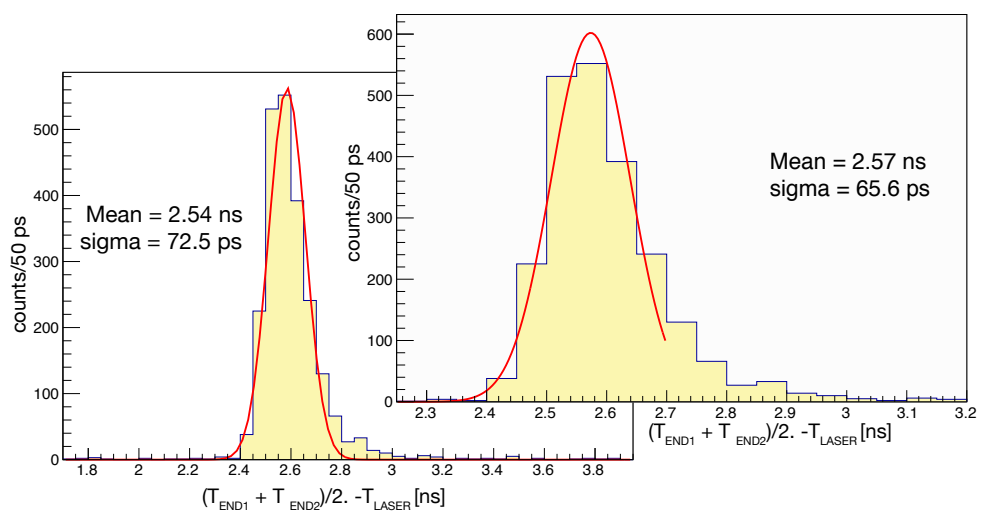

Figure 9. Each end of the Strip SiPM is connected to a NINO input that is then digitised by the SAMPET system. The average of the times measured at each end is subtracted from the time of the laser pulse and shown in this histogram. A Gaussian fit is made to the histogram with the $\sigma$ of this fit quoted as the SPTR. It is clear that the fit is influenced by the late tail of hits. If the range of the fit is reduced (as shown in the inset) the $\sigma$ of the fit in this case reduces from $72.5 \mathrm{ps}$ to $65.6 \mathrm{ps}$. All SPTR values quoted in this paper are obtained from a fit of the full range of the histogram.

light could also be moved vertically to select a particular strip and horizontally from one end of the SiPM to the other. In figure 10 we show the SPTR measured for all 16 strips for three different voltages. Clearly the SPTR improves as the voltage is increased; also it appears that some strips are better than others; however, since we apply the same voltage for all strips, this apparent difference may be caused by a difference in breakdown voltage for individual strips.

In figure 11 the spot of the laser light is scanned from one end of a strip to the other. The time of the signal from each end is shown - and also the average of the time measurement. The 


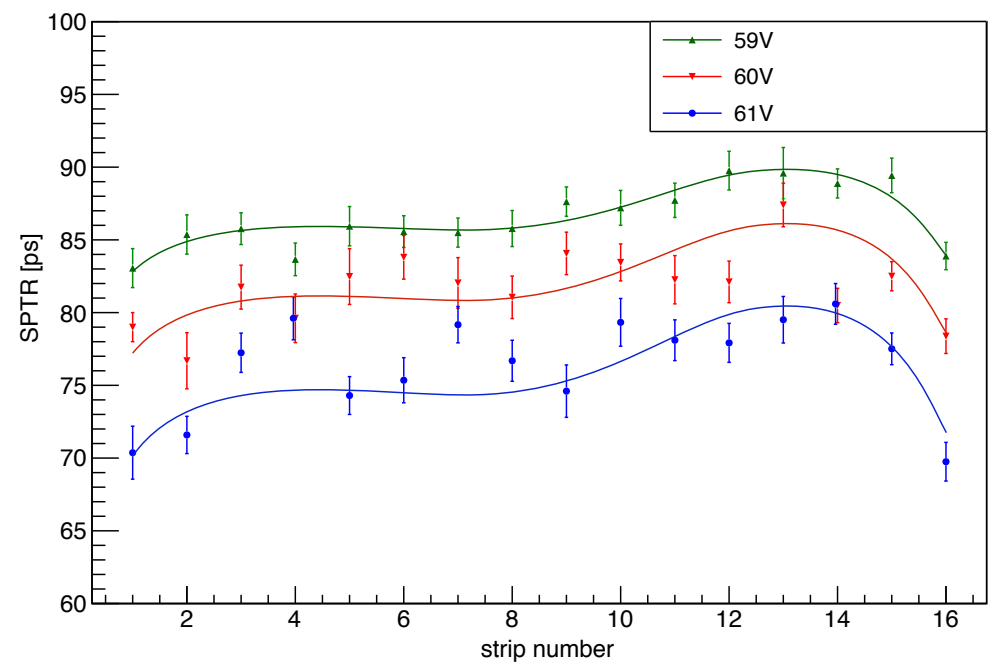

Figure 10. The single photon time resolution (SPTR) measured for all 16 strips making an array for three different applied voltages. The lines are drawn to guide the eye.

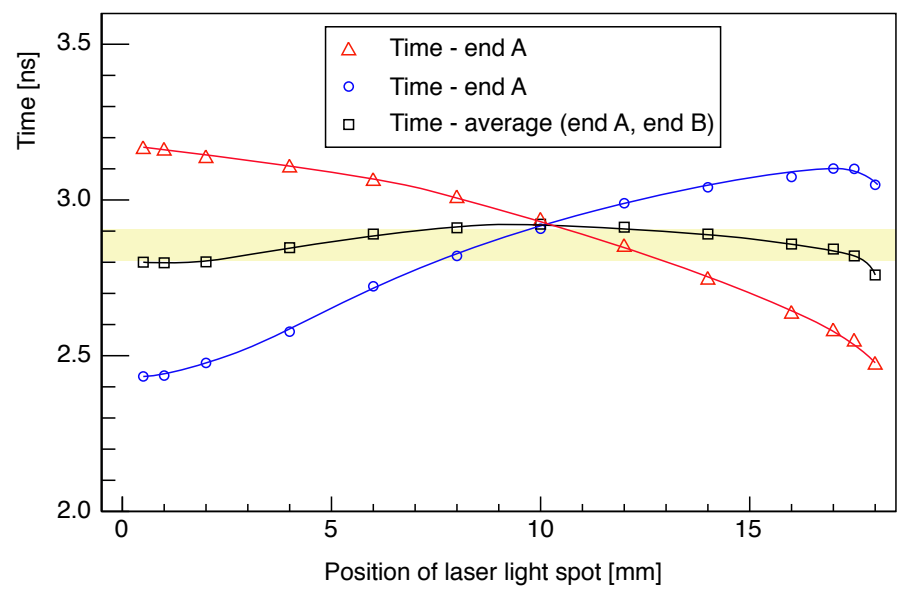

Figure 11. As the spot of the laser light is moved along the strip, the time of the signal from each end will change; however the average of the times of the signal from each end should be a constant value. The highlighted band shows that the average value varies by $100 \mathrm{ps}$ along the length of the strip.

average varies by $\sim 100 \mathrm{ps}$; this variation can be corrected. In figure 12 we show a measurement of the position resolution obtained from time difference. Since the total time difference is $1.4 \mathrm{~ns}$ (see figure 6) the $\sigma$ of the time difference can be converted into a distance. The value that corresponds to a $\sigma$ of $1 \mathrm{~mm}(77 \mathrm{ps})$ is shown on the figure.

\section{Discussion}

As we have presented in this paper we can achieve a single photon time resolution around $75 \mathrm{ps}$ over the full surface of the array of strip SiPMs. Since the SiPM is temperature sensitive, we need to keep it cool and at a fixed temperature even in the situation that the front-end readout electronics generates significant heat. In this example we transport the signals $\sim 5 \mathrm{~cm}$ with traces on the PCB 


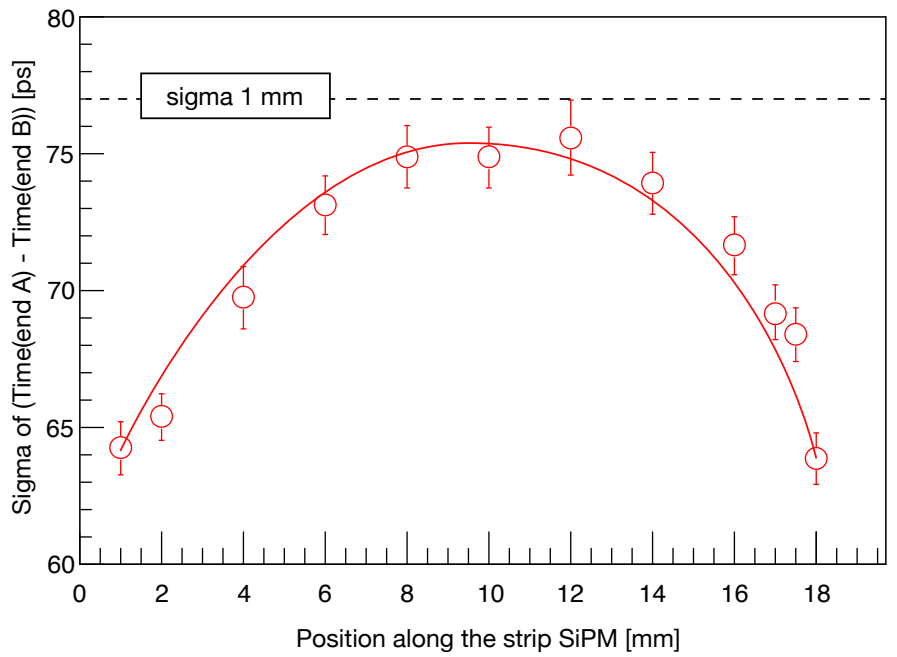

Figure 12. The position along the length of the strip can be calculated from the time difference between the signals from each end of the strip. The $\sigma$ of this time difference spectra is a measure of the position resolution; this is shown in this plot versus the position of the laser spot along the length of the strip. A $\sigma$ of $77 \mathrm{ps}$ corresponds to a position resolution $(\sigma)$ of $1 \mathrm{~mm}$. The line is to guide the eye.

and still obtain relatively good timing. We will add a cooling system to absorb the heat from the front-end electronics that also keeps the SiPM array at a fixed temperature.

\section{Acknowledgments}

This work has been supported by the National Research Foundation of Korea, Grant Agreements 2019R1I1A3A01056616, 2019K1A3A1A25000088, and 2019K1A3A1A79112972.

\section{References}

[1] V. Saveliev and V. Golovin, Novel type of avalanche photodetector with Geiger mode operation, Nucl. Instrum. Meth. A 442 (2000) 223.

[2] Z. Sadygov, Avalanche detector, Russian patent RU2102820, application from October 10 (1996), published in January 20 (1998).

[3] K. Doroud, A. Rodriguez, M.C.S. Williams, K. Yamamoto, A. Zichichi and R. Zuyeuski, Systematic study of new types of Hamamatsu MPPCs read out with the NINO ASIC, Nucl. Instrum. Meth. A 753 (2014) 149 [INSPIRE].

[4] K. Doroud, E. Auffray, P. Jarron, T. Meyer and P. Lecoq, Differential-readout: the technique to optimise timing in a monolithic MPPC array, Nucl. Instrum. Meth. A 717 (2013) 5 [INSPIRE].

[5] K. Doroud, M.C.S. Williams and K. Yamamoto, The Strip Silicon Photo-Multiplier: an innovation for enhanced time and position measurement, Nucl. Instrum. Meth. A 853 (2017) 1 [INSPIRE].

[6] C. Piemonte and A. Gola, Overview on the main parameters and technology of modern Silicon Photomultipliers, Nucl. Instrum. Meth. A 926 (2019) 2.

[7] F. Anghinolfi et al., NINO: an ultra-fast and low-power front-end amplifier/discriminator ASIC designed for the multigap resistive plate chamber, Nucl. Instrum. Meth. A 533 (2004) 183 [INSPIRE]. 
[8] D. Breton, C. Cheikali, E. Delagnes, J. Maalmi, P. Rusquart and P. Vallerand, Fast electronics for particle Time-Of-Flight measurement, with focus on the SAMPIC ASIC, Nuovo Cim. C 43 (2020) 7 [INSPIRE].

[9] P. Eckert et al., Characterisation studies of Silicon Photomultipliers, Nucl. Instrum. Meth. A 620 (2010) 217.

[10] D. Renker and E. Lorenz, Advances in solid state photon detectors, 2009 JINST 4 P04004 [INSPIRE]. 